\title{
Structure and microhardness of the three-component Ni-Mn-In alloy after different modes of thermal cycling treatment
}

\author{
Yu. V. Kaletina ${ }^{\dagger}$ E. D. Greshnova, A. Yu. Kaletin \\ †kaletina@imp.uran.ru
}

Mikheev Institute of Metal Physics, Ural Branch of RAS, 18 S. Kovalevskoi st., 620137, Yekaterinburg, Russia

The influence of various modes of thermal cycling treatment on the microstructure and properties of ferromagnetic alloy $\mathrm{Ni}_{47} \mathrm{Mn}_{42} \mathrm{In}_{11}$ was studied. The modes of thermal cycling treatment differed in the heating temperature $(363-573 \mathrm{~K})$ and holding time, the cooling temperature being $77 \mathrm{~K}$ for all modes. The number of heating-cooling cycles was varied from 1 to 30. The microstructure of $\mathrm{Ni}_{47} \mathrm{Mn}_{42} \mathrm{In}_{11}$ alloy in the initial state and after thermal cycling treatment was studied by optical metallography and scanning electron microscopy. A magnetic structural transition is observed in the alloy during cooling from the high-temperature region at a temperature about $T \approx 300-310 \mathrm{~K}$. In the initial state, the structure of the alloy has two phases and consists of the L2 ${ }_{1}$ phase and martensite. The martensite crystals are grouped in packets of $80-150 \mu \mathrm{m}$ width, which are misoriented relative to each other by angles of 60 or 120 degrees. The boundaries of the coarse grains are predominantly smooth in the initial state. Structural studies showed that after thermal cycling treatment the martensitic crystals reached the grain boundaries and deformed them, and boundaries of a serrate form appeared. Micro-X-ray spectral analysis did not reveal precipitates of the second phases along the grain boundaries after thermal cycling treatment. The stress level was estimated after annealing and subsequent thermal cycling treatment by electron back scattered diffraction method. It was shown that with an increase in the number of heating-cooling cycles the stress level in the material increased. After the thermal cycling treatment the microhardness of the investigated alloy increased. It was shown that there was no unequivocal dependence of the microhardness value on the temperature of heating upon the thermocycling processes. The maximum increment in microhardness was detected after 20 cycles of treatment by in the $473 \mathrm{~K} \leftrightarrow 77 \mathrm{~K}$ mode.

Keywords: ferromagnetic alloy, structure, martensite transformation, thermal cycling, microhardness.

\section{Структура и микротвердость трехкомпонентного сплава Ni-Mn-In после различных режимов термоциклической обработки}

\author{
Калетина Ю. В. ${ }^{\dagger}$, Грешнова Е.Д., Калетин А. Ю. \\ Институт физики металлов им. М.Н. Михеева УрО РАН, ул. С.Ковалевской 18, 620137, Екатеринбург, Россия
}

Исследовано влияние различных режимов термоциклической обработки на микроструктуру и свойства ферромагнитного сплава $\mathrm{Ni}_{47} \mathrm{Mn}_{42} \mathrm{In}_{11}$. Режимы термоциклической обработки отличались температурой нагрева (363-573 K) и временем выдержки, температура охлаждения для всех режимов - 77 К. Количество циклов нагрев-охлаждение варьировали от 1 до 30. Методами оптической металлографии и сканирующей электронной микроскопии изучали микроструктуру сплава $\mathrm{Ni}_{47} \mathrm{Mn}_{42} \mathrm{In}_{11}$ в исходном состоянии и после термоциклической обработки. При охлаждении из высокотемпературной области в сплаве при $T \approx 300-310 \mathrm{~K}$ наблюдается магнитоструктурный переход. В исходном состоянии структура сплава двухфазная, состоит из L2 1 -фазы и мартенсита. Кристаллы мартенсита сгруппированы в пакеты шириной 80-150 мкм, которые разориентированы относительно друг друга на угол 60 или 120 градусов. В исходном состоянии границы крупных зерен преимущественно ровные. Структурные исследования показали, что после термоциклической обработки мартенситные кристаллы при достижении границ зерен часто деформируют их, и появляются границы зубчатой формы. Микрорентгеноспектральный анализ после термоциклической обработки не выявил выделений вторых фаз по границам зерен. Методом дифракции обратно отраженных электронов была проведена оценка уровня напряжений после отжига и последующей термоциклической обработки. Показано, что с увеличением числа циклов нагрев-охлаждение уровень напряжений в материале возрастает. После термоциклической обработки повышается микротвердость исследованного сплава. Показано, что температура нагрева при термоциклической обработке неоднозначно влияет на величину микротвердости. Максимальный прирост микротвердости обнаружен после 20 циклов обработки по режиму $473 \mathrm{~K} \leftrightarrow 77 \mathrm{~K}$.

Ключевые слова: ферромагнитный сплав, структура, мартенситное превращение, термоциклическая обработка, микротвердость. 


\section{1. Введение}

Одной из задач физического материаловедения является создание новых металлических материалов и разработка технологий их практического применения. Сплавы Гейслера можно выделить в особый класс перспективных «интеллектуальных» материалов, обладающих функциональными свойствами. Интерес к исследованиям структуры и свойств ферромагнитных сплавов Гейслера возрастает. Это связано с тем, что они обладают сочетанием целого ряда свойств и эффектов, таких как эффект памяти формы, магнетокалорический эффект, гигантские магнетосопротивление и магнетодеформации и другие. Достижения последних лет [1-12] в области изучения поведения различных по составу сплавов Гейслера под воздействием магнитного поля, температуры, давления показали реальную возможность использования их в качестве сенсоров, актюаторов, в технологии магнитного охлаждения, а также в медицинских целях.

Трехкомпонентные сплавы Гейслера на основе систем $\mathrm{Ni}-\mathrm{Mn}-\mathrm{Z}$ (Z = In, Sn, Sb) представляют отдельную группу. В этих системах нестехиометрических составов, как показали многочисленные исследования [1-3, 7-11], наблюдается сложная последовательность структурнофазовых превращений. В наших работах [9-11] была исследована серия сплавов на основе Ni-Mn-In. Показано, как при замещении атомов никеля атомами марганца изменяется картина фазовых магнитных и структурных переходов.

В работе [13] представлены результаты по влиянию термоциклирования на структуру и свойства серии сплавов $\mathrm{Ni}_{47-x} \mathrm{Mn}_{42+x} \mathrm{In}_{11}(0 \leq x \leq 2)$. Наибольший интерес представляет сплав нестехиометрического состава $\mathrm{Ni}_{47} \mathrm{Mn}_{42} \mathrm{In}_{11}$, у которого температура структурного мартенситного превращения близка к температуре магнитного перехода и к комнатной температуре. Было установлено [13], что сплав такого состава в большей степени чувствителен в термоциклической обработке по сравнению с другими сплавами из этой серии. Было показано [13], что после многократных циклов нагрева в однофазную область существования парамагнитной L2 фазы и охлаждения (до температуры жидкого азота) уровень микротвердости в сплаве $\mathrm{Ni}_{47} \mathrm{Mn}_{42} \mathrm{In}_{11}$ существенно повышается по сравнению с микротвердостью двух других сплавов $\mathrm{Ni}_{45} \mathrm{Mn}_{44} \mathrm{In}_{11}$ и $\mathrm{Ni}_{46} \mathrm{Mn}_{43} \mathrm{In}_{11}$. Поэтому целью настоящей работы являлось исследование влияния разных режимов термоциклической обработки на особенности структуры и свойства сплава $\mathrm{Ni}_{47} \mathrm{Mn}_{42} \mathrm{In}_{11}$.

\section{2. Материал и методика исследования}

Исследовали тройной сплав $\mathrm{Ni}_{47} \mathrm{Mn}_{42} \mathrm{In}_{11}$, который был выплавлен методом электродуговой плавки в атмосфере аргона. После выплавки проводили гомогенизирующий отжиг при температуре $1123 \mathrm{~K}$ в течение 24 ч с последующим охлаждением с печью. Затем вырезали образцы для термообработки.

После отжига проводили термоциклическую обработку (ТЦО) по разным режимам. Режимы ТЦО отли- чались температурой нагрева $T_{h}$ и временем выдержки, температура охлаждения $T_{\text {cool }}$ для всех режимов была постоянной. В ходе каждого термического цикла одну группу образцов нагревали до температуры $T_{h 1}=363 \mathrm{~K}$ (время выдержки -5 мин), другую группу - до $T_{h 2}=473 \mathrm{~K}$ (время выдержки - 10 мин), третью группу - до $T_{h 3}=573 \mathrm{~K}$ (время выдержки - 10 мин). После нагрева все образцы охлаждали до температуры жидкого азота $T_{\text {cool }}=77 \mathrm{~K}$. Время выдержки при $T_{\text {cool }}$ составило 5, 30 и 10 мин, соответственно для каждой группы образцов. Схема режимов ТЦО приведена на рис. 1. Количество циклов нагрев-охлаждение варьировали от 1 до 20-30 в зависимости от режима ТЦО. Исследования микроструктуры сплава $\mathrm{Ni}_{47} \mathrm{Mn}_{42} \mathrm{In}_{11}$ и измерения его микротвердости проводились при комнатной температуре.

Структурные исследования выполнены на оптическом микроскопе «Neophot-30» на шлифах после травления. Электронно-микроскопические исследования и построение микрокарт кристаллографических ориентаций проводили на сканирующем электронном микроскопе «Quanta-200» с локальным микрорентгеноспектральным анализом. Кристаллографические ориентации внутризеренной области определяли методом дифракции обратно отраженных электронов (EBSD). Сканирование шлифа осуществлялось с шагом 0,15 мкм. Измерения микротвердости проводили на шлифах после травления на приборе ПМТ-3 по стандартной методике при нагрузке на индентер $0,5 \mathrm{H}$, и определяли как среднее арифметическое из 20 параллельных измерений, выполненных при условии сохранения геометрии отпечатка (пирамидки Виккерса).

\section{3. Результаты и их обсуждение}

Исследуемый сплав при высоких температурах имеет кубическую кристаллическую решетку типа L2. При охлаждении в сплаве наблюдается магнитный переход высокотемпературной L2 1 фазы из парамагнитного в ферромагнитное состояние, температура Кюри $T_{\mathrm{CA}} \approx 310 \mathrm{~K}[11]$. При дальнейшем охлаждении при температуре $T_{\mathrm{M}} \approx 300 \mathrm{~K}$ происходит мартенситное превращение. Следует отметить, что в сплаве $\mathrm{Ni}_{47} \mathrm{Mn}_{42} \mathrm{In}_{11}$ температура Кюри аустенита близка к температуре мартенситной точки $T_{\mathrm{CA}} \approx T_{\mathrm{M}}$. Поэтому можно считать,

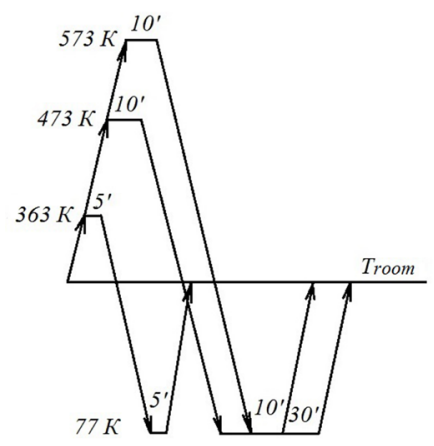

Рис. 1. Схема термоциклической обработки по различным режимам образцов из сплава $\mathrm{Ni}_{47} \mathrm{Mn}_{42} \mathrm{In}_{11}$.

Fig. 1. Scheme of thermal cycling treatment for various modes of $\mathrm{Ni}_{47} \mathrm{Mn}_{42} \mathrm{In}_{11}$ alloy samples. 
что наблюдается магнитоструктурный переход. Таким образом, при комнатной температуре в исходном состоянии структура сплава $\mathrm{Ni}_{47} \mathrm{Mn}_{42} \mathrm{In}_{11}$ двухфазная, состоящая из L2 1 фазы и мартенсита (рис. 2a, b). Кристаллы мартенсита сгруппированы в пакеты шириной от 80 до 150 мкм, которые разориентированы относительно друг друга на угол 60 или 120 градусов. Металлографические и электронно-микроскопические исследования показали, что внутри пакетов тонкие мартенситные пластины с плоскими границами разделов располагаются преимущественно параллельно друг другу. Ширина мартенситных пластин - от 300 нм до 2,0-2,7 мкм. Микроструктура поликристаллическая, как правило с ровными границами исходных крупных зерен. Кристаллы мартенсита имеют разную морфологию. Кроме пластинчатых кристаллов в структуре выявлены кристаллы клиновидной формы (рис. 2b). Клиновидные кристаллы указаны стрелками на рис. 2b. Разная морфология мартенситных кристаллов, по-видимому, обусловлена условиями структурного превращения, протекающего в среде с высокими упругими свойствами.

После термоциклической обработки сплава $\mathrm{Ni}_{47} \mathrm{Mn}_{42} \mathrm{In}_{11}$ существенных изменений в структуре не выявлено - наряду с высокотемпературной фазой

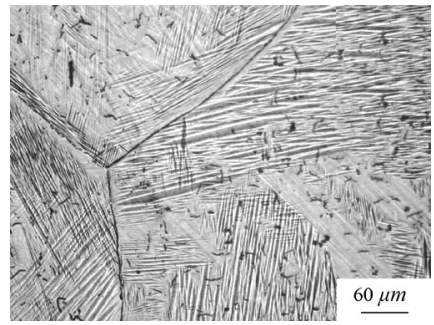

a

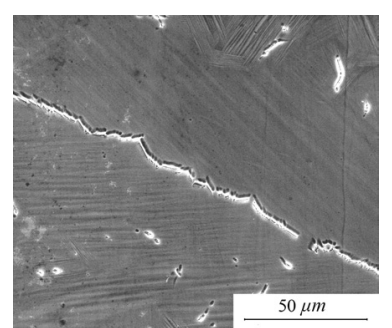

C

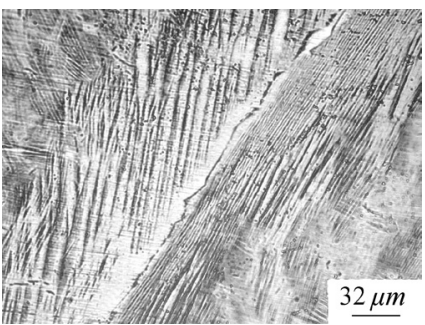

e

Рис. 2. Микроструктура сплава $\mathrm{Ni}_{47} \mathrm{Mn}_{42} \mathrm{In}_{11}:$ в исходном состоянии (a, b); после 20 циклов нагрев-охлаждение с температурой нагрева $T_{h 1}=363 \mathrm{~K}(\mathrm{c}) ; T_{h 2}=473 \mathrm{~K}(\mathrm{~d}) ;$ после 4 циклов нагрев-охлаждение с температурой нагрева $T_{h 3}=573 \mathrm{~K}(\mathrm{e})$.

Fig. 2. Microstructure of $\mathrm{Ni}_{47} \mathrm{Mn}_{42} \mathrm{In}_{11}$ alloy: initial condition (a, b); after 20 heating-cooling cycles with heating temperature $T_{h 1}=363 \mathrm{~K}(\mathrm{c}) ; T_{h 2}=473 \mathrm{~K}$ (d); after 4 heating-cooling cycles with heating temperature $T_{h 3}=573 \mathrm{~K}(\mathrm{e})$.
L2 1 присутствуют кристаллы мартенсита. Однако после ТЦО сплава $\mathrm{Ni}_{47} \mathrm{Mn}_{42} \mathrm{In}_{11}$ заметно изменяется строение границ зерен (см. рис. 2c-2e). Границы приобретают неровную, зубчатую форму. В отдельных случаях растущие кристаллы мартенсита при достижении границы проходят сквозь нее, местами границы разрушаются и образуются трещины. Такая картина наблюдалась после всех трех режимов ТЦО. Вероятно, в материале происходит рост уровня напряжений. С увеличением числа циклов нагрева и охлаждения зубчатых границ становится больше.

Микрорентгеноспектральный анализ после ТЦО показал отсутствие каких-либо выделений вторых фаз на границах зерен сплава $\mathrm{Ni}_{47} \mathrm{Mn}_{42} \mathrm{In}_{11}$. В спектре с участка границы зерна, отмеченного крестом на рис. 3а, присутствуют только линии элементов, входящих в исследуемый сплав.

Представляло интерес оценить уровень напряжений в сплаве после ТЦО и сравнить его с исходным состоянием. Современные виды микроскопии для исследования кристаллических материалов позволяют выявлять особенности микроструктуры [17]. Для изучения процессов, происходящих в твердых телах, например, мартенситного превращения, применяется автоматический

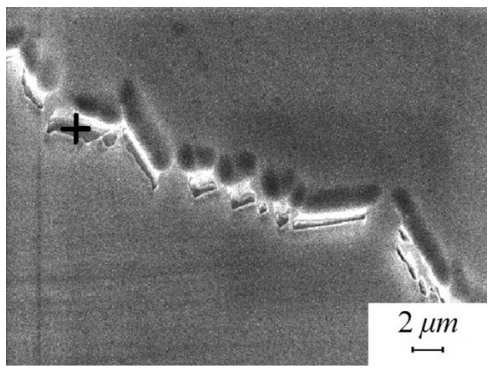

a

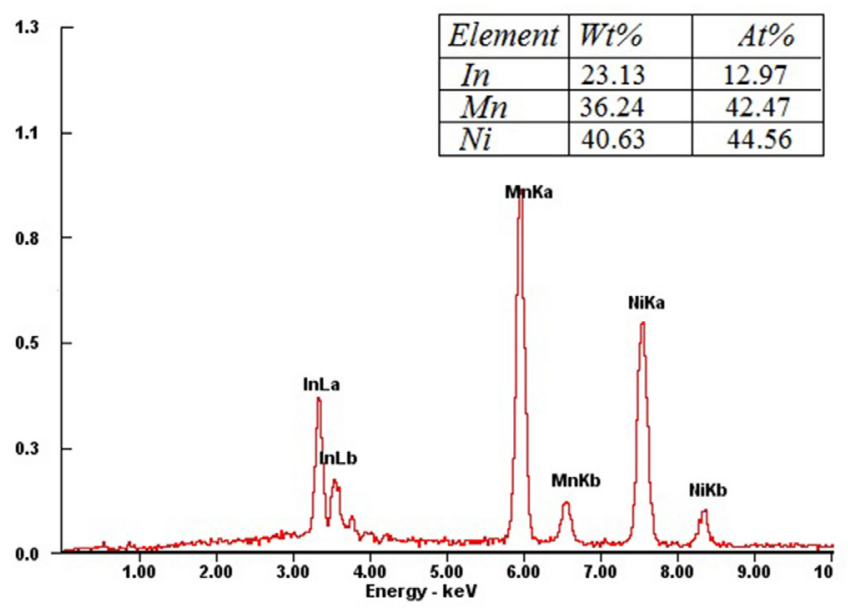

b

Рис. 3. Микрорентгеноспектральный анализ границы зерна сплава $\mathrm{Ni}_{47} \mathrm{Mn}_{42} \mathrm{In}_{11}$ после 20 циклов нагрев-охлаждение с температурой нагрева $T_{h 1}=363 \mathrm{~K}$ : микроструктура (a); спектр c анализируемого участка границы зерна, обозначенного крестом (b).

Fig. 3. Micro-X-ray spectral analysis of the grain boundary of the alloy $\mathrm{Ni}_{47} \mathrm{Mn}_{42} \mathrm{In}_{11}$ after 20 heating-cooling cycles with heating temperature $T_{h 1}=363 \mathrm{~K}$ : microstructure (a); spectrum from the analyzed section of the grain boundary marked by a cross (b). 
анализ картин дифракции обратно рассеянных электронов. C помощью EBSD-анализа были получены чернобелые картины качества изображения (image quality), снятые на образцах из сплава $\mathrm{Ni}_{47} \mathrm{Mn}_{42} \mathrm{In}_{11}$ в исходном состоянии (рис. 4) и после двадцати циклов нагрев-охлаждение по схеме $473 \mathrm{~K} \leftrightarrow 77 \mathrm{~K}$ (рис. 5).

На рис. 4 приведены картина качества изображения (рис. 4а) и карта кристаллографической ориентировки кристаллов мартенсита (рис. 4b) сплава $\mathrm{Ni}_{47} \mathrm{Mn}_{42} \mathrm{In}_{11}$ в исходном состоянии. Представлены фрагменты мартенситных пакетов, состоящие из кристаллов мартенсита пластинчатой и клиновидной формы различной ширины (0,5-3 мкм). Более тонкие кристаллы мартенсита не идентифицированы.

Картины качества изображения определяются возможностью расшифровки линий Кикучи, что может быть мерой степени напряженности материала. С увеличением деформации решетки, происходит размытие Кикучи линий, что значительно снижает качество сканирования [18]. Возникает сложность корректного определения ориентировки кристаллитов, увеличивается удельная доля точек на ориентационной карте, от которых не произошло формирование линий Кикучи [17]. В свою очередь на картине качества изображения области, в которых расшифровка линий Кикучи затруднена или невозможна, окрашиваются в темно-серый или черный цвет. В случае, когда расшифровка происходит полностью, областям присваивается белый цвет.

После 20 циклов нагрев-охлаждение $473 \mathrm{~K} \leftrightarrow 77 \mathrm{~K}$ уровень напряжений в материале растет. В результате картина качества изображения имеет более низкий контраст (рис. 5a), а EBSD-микрокарта характеризуется повышенным уровнем размытия субструктурных элементов и границ мартенситных кристаллов (рис. 5b). В семь раз увеличилась удельная доля точек, от которых не произошло формирование линий Кикучи, по сравнению с исходным состоянием, что затрудняет определение точных кристаллографических ориентировок мартенсита.

Таким образом, увеличение удельной доли точек, от которых не произошло формирование линий Кикучи соответствует виду EBSD- ориентационной карты. Снижение качества расшифровки линий Кикучи косвенно указывает на увеличение степени деформации кристаллической решетки материала. Повышение внутренних напряжений в сплаве $\mathrm{Ni}_{47} \mathrm{Mn}_{42} \mathrm{In}_{11}$ после ТЦО подтверждается ростом значений микротвердости.

На рис. 6 показаны средние значения микротвердости $\mathrm{Ni}_{47} \mathrm{Mn}_{42} \mathrm{In}_{11}$ в исходном состоянии и после термоциклической обработки по разным режимам. После ТЦО по всем трем режимам наблюдали увеличение микротвердости. Однако наибольший прирост значений микротвердости обнаружен после 20 циклов нагрева до $T_{h 2}=473 \mathrm{~K}$ и охлаждения до $77 \mathrm{~K}$.

Была найдена разность значений микротвердости $\Delta H V$ в исходном состоянии и после ТЦО по различным

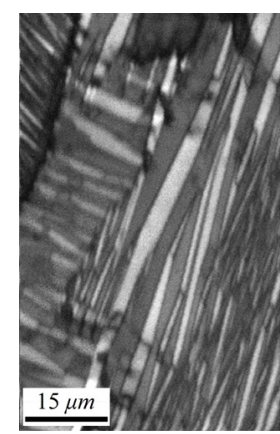

a

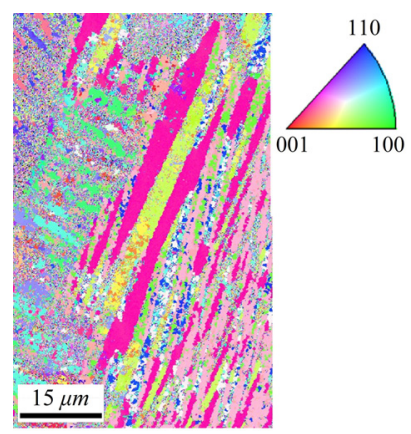

$\mathrm{b}$
Рис. 4. Картина качества изображения (а) и EBSD-микрокарта ориентировок кристаллов мартенсита (b) сплава $\mathrm{Ni}_{47} \mathrm{Mn}_{42} \mathrm{In}_{11}$ после отжига при температуре 1123 К в течение 24 ч.

Fig. 4. Image quality (a) and crystallographic orientation micrograph of martensite crystals (b) of $\mathrm{Ni}_{47} \mathrm{Mn}_{42} \mathrm{In}_{11}$ alloy after annealing at temperature $1123 \mathrm{~K}$ for $24 \mathrm{~h}$.

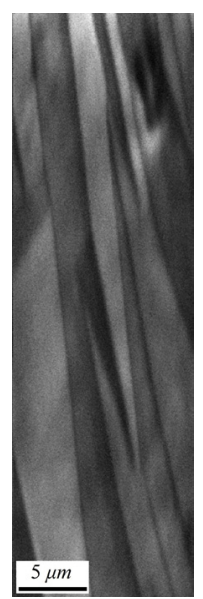

a b

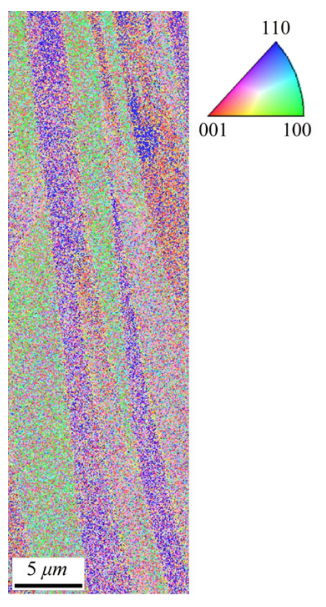

Рис. 5. Картина качества изображения (a); EBSD- ориентационная карта кристаллов мартенсита сплава $\mathrm{Ni}_{47} \mathrm{Mn}_{42} \mathrm{In}_{11}$ после 20 циклов нагрев-охлаждение $473 \mathrm{~K} \leftrightarrow 77 \mathrm{~K}$ (b).

Fig. 5. Image quality (a); crystallographic orientation micrograph of martensite crystals of $\mathrm{Ni}_{47} \mathrm{Mn}_{42} \mathrm{In}_{11}$ alloy after 20 heating-cooling cycles $473 \mathrm{~K} \leftrightarrow 77 \mathrm{~K}(\mathrm{~b})$.

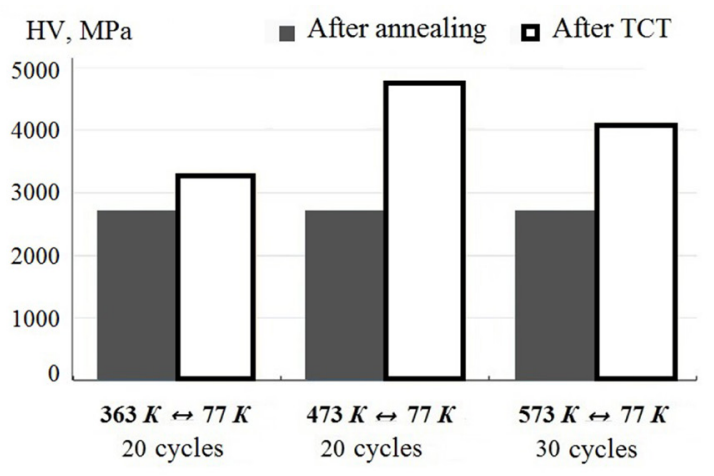

Рис. 6. Влияние режимов термоциклической обработки на микротвердость сплава $\mathrm{Ni}_{47} \mathrm{Mn}_{42} \mathrm{In}_{11}$.

Fig. 6. Effect modes of thermal cycling on the microhardness of the $\mathrm{Ni}_{47} \mathrm{Mn}_{42} \mathrm{In}_{11}$ alloy. 
режимам. Наибольшая разность $\Delta H V_{473}=2000$ МПа была обнаружена после обработки по режиму $473 \mathrm{~K} \leftrightarrow 77 \mathrm{~K}$ (20 циклов). После обработки по режиму $573 \mathrm{~K} \leftrightarrow 77 \mathrm{~K}$ (30 циклов) разность $\Delta H V_{573}=1300$ МПа. Для режимов ТЦО $363 \mathrm{~K} \leftrightarrow 77 \mathrm{~K}$ (20 циклов) разность значений микротвердости самая низкая и составила $\Delta H V_{363}=600$ МПа. Проведенные исследования показали, что не наблюдается однозначного влияния температуры нагрева при ТЦО на уровень микротвердости исследуемого сплава.

\section{4. Заключение}

Исследовано влияние разных режимов термоциклической обработки на особенности структуры и микротвердость сплава $\mathrm{Ni}_{47} \mathrm{Mn}_{42} \mathrm{In}_{11}$. Показано, что многократные циклы нагрева в область высокотемпературной L2, фазы и охлаждения до низких температур влияют на структуру, уровень напряжений и микротвердость сплава $\mathrm{Ni}_{47} \mathrm{Mn}_{42} \mathrm{In}_{11}$.

После термоциклирования мартенситные кристаллы деформируют и разрушают границы зерен, что приводит к охрупчиванию сплава. Не обнаружено выделения частиц вторых фаз по границам зерен после ТЦО.

Оценка уровня напряжений методом EBSD анализа показала, что после разных режимов термоциклической обработки с увеличением числа циклов нагрев - охлаждение увеличивается уровень напряжений в материале.

После термоциклической обработки микротвердость возрастет. Температура нагрева при ТЦО неоднозначно влияет на величину микротвердости. Максимальное значение микротвердости получено после ТЦО по режиму $473 \mathrm{~K} \leftrightarrow 77$ К (20 циклов).

Благодарность/Acknowledgements. Электронно-микроскопические исследования проведены в отделе электронной микроскопии ЦКП «Испьтательный иентр нанотехнологий и перспективных материалов» ИФМ УрО РАН. Работа выполнена в рамках государственного задания ФАНО России (тема «Структура», № 01201463331) при частичной поддержке РФФИ (проект № 16-03-00043).

\section{Литература/References}

1. Y. Sutou, Y. Imano, N. Koeda, Y. Omori, R. Kainuma, K. Ishida, K. Oikawa. Appl. Phys. Lett. 85(9), 4358-4360 (2004), doi:10.1063/1.1808879

2. T. Krenke, E. Duman, M. Acet, E. F. Wassermann, X. Moya, L. Manosa. Planes A. Nat. Mater. 4, 450-454 (2005), doi:10.1038/nmat1395

3. V.D. Buchel'nikov, A.N. Vasiliev, V.V. Koledov, V.V. Hovaylo, S. V. Taskaev, V.G. Shavrov. Phys. Usp. 49, 871 (2006). (in Russian) [В.Д. Бучельников, А.Н. Васильев, В.В. Коледов, В.В. Ховайло, С. В. Таскаев, В.Г. Шавров. УФН 176, 900 (2006).] doi: 10.1070/PU2006v049n08ABEH006081
4. K. Oikawa, W. Ito, Y. Imano, Y. Sutou, R. Kainuma, K. Ishida, S. Okamoto, O. Kitakami, T. Kanomata. Appl. Phys. Lett. 88, 122507 (2006), doi: 10.1063/1.2187414

5. W. Ito, Y. Imano, R. Kainuma, Y. Sutou, K. Oikawa, K. Ishida. Metall. Mater. Trans. A. 38A, $759-766$ (2007), doi: 10.1007/s11661-007-9094-9

6. V.M. Schastlivtsev, Yu. V. Kaletina, E.A. Fokina. Martensitic transformation in a magnetic field. UB RAS. (2007) 322 p. (in Russian) [В. М. Счастливцев, Ю.В. Калетина, Е. А. Фокина. Мартенситное превращение в магнитном поле. УрО РАН, Екатеринбург. 2007. 322 c.]

7. T. Graf, C. Felser, S. S. P. Parkin. Prog. Solid State Chem. 39, 1 -51 (2011), doi: 10.1016/j.progsolidstchem.2011.02.001

8. V.D.Buchel'nikov, V. V.Sokolovskiy.Phys. Metals Metallogr. 112(7), 633 (2011), doi: 10.1134/S0031918X11070052

9. Yu. V. Kaletina, V.M. Schastlivtsev, A.V. Korolev, E. A. Fokina. Phys. Metals Metallogr. 113, 1029 (2012). (in Russian) [Ю.В. Калетина, В.М. Счастливцев, А.В. Королев, Е.А. Фокина. ФММ. 113(11), 1086 (2012).] doi: 10.1134/S0031918X12110129

10. Yu. V. Kaletina, E. G. Gerasimov, V.M. Schastlivtsev, E. A. Fokina, P.B. Terentyev. Phys. Metals Metallogr. 114, 838 (2013). (in Russian) [Ю.В. Калетина, Е.Г. Герасимов, В.М. Счастливцев, Е.А. Фокина, П.Б. Терентьев. ФММ. 114(10), 911 (2013).] doi: 10.1134/S0031918X13100050

11. Yu. V. Kaletina, E. G. Gerasimov. Phys. Solid State. 56, 1634 (2014). (in Russian) [Ю.В. Калетина, Е. Г. Герасимов. ФTT. 56, 1583 (2014).] doi: 10.1134/S1063783414080101

12. H. Yan, B. Yang, Y. Zhang, Z. Li, C. Esling, X. Zhao, L.Zuo. Acta Mat. 111, $75-84$ (2016), doi: 10.1016/j. actamat.2016.03.049

13. Yu. V. Kaletina, E.D. Efimova, E.G. Gerasimov, A. Yu. Kaletin. Tech. Phys. 61, 1894 (2016). (in Russian) [Ю.В. Калетина, Е.Д. Ефимова, Е.Г. Герасимов, А.Ю. Калетин. ЖТФ. 86(1), 155 (2016).] doi: 10.1134/S1063784216120197

14. H. Warlimont, L. Delaey. Martensitic transformations in copper-silver- and gold-base alloys. Pergamon Press. (1980) 208 p.

15. A. N. Vasiliev, V. D. Buchel'nikov, T. Takagi, V. V. Hovaylo, E. I. Estrin. Phys. Usp. 46, 559 (2003). (in Russian) [А.Н. Васильев, В.Д. Бучельников, Т. Такаги, В. В. Ховайло, Э. И. Эстрин. УФН. 173, 577 (2003).] doi: 10.1070/PU2003v046n06ABEH001339

16. A. Planes, L. Manosa, M. Acet. J. Phys.: Condens. Matter. 21, 233201 - 29 (2009), doi: 10.1088/0953-8984/21/23/ 233201

17. S. Yu. Mironov, V.N. Danilenko, M.M. Myshlyaev, A. V. Korneva. Phys. Solid State. 47(7), 1258 (2005). (in Russian) [С.Ю. Миронов, В.Н. Даниленко, М.М. Мышляев, А.В. Корнева. ФТТ. 47(7), 1217 (2005).] doi: 10.1134/1.1992602

18. A. J. Wilkinson, D. J. Dingley. Acta Metall. Mater. 39(12), 3047 - 3055 (1991), doi: 10.1016/0956 - 7151(91)90037-2 